\title{
Botulinum Toxin Injection for Managing Sleep Bruxism
}

\author{
Seurin Kim, DDS, MSD ${ }^{1}$, Keun jeong Park, DDS ${ }^{1}$, Young joo Shim, DDS, MSD, PhD², Seong Taek Kim, DDS, MSD, PhD ${ }^{1}$ \\ ${ }^{1}$ Department of Orofacial Pain and Oral Medicine, Yonsei University College of Dentistry, Seoul, Korea \\ ${ }^{2}$ Department of Orofacial Pain and Oral Medicine, Daejeon Dental Hospital, College of Dentistry, Wonkwang University, Daejeon, Korea
}

\footnotetext{
Received: June 1, 2018

Revised: July 2, 2018

Accepted: September 18, 2018

Correspondence

Seurin Kim, DDS, MSD

Department of Orofacial Pain and

Oral Medicine, Yonsei University

College of Dentistry,

50-1 Yonsei-ro, Seodaemun-gu,

Seoul 03722, Korea

Tel +82-2-2228-8877

Fax +82-2-393-5673

E-mail SERENE86@yuhs.ac

ORCID

Seurin Kim

https://orcid.org/0000-0003-0844-3765

Keun jeong Park

https://orcid.org/0000-0003-4559-9398

Young joo Shim

https://orcid.org/0000-0001-7514-5974

Seong Taek Kim

https://orcid.org/0000-0001-9506-5103
}

Botulinum toxin (BoNT), a potent neurotoxin that reversibly blocks presynaptic acetylcholine release, has been applied successfully to treat severe sleep bruxism (SB). However, little work has been reviewed on the effectiveness of BoNT and its injection protocol such as drug dose and injection sites in masticatory muscles. Therefore, we reviewed recent studies on application of BoNT in SB patients.

Sleep Med Res 2018;9(2):73-76

Key Words Botulinum toxins, Sleep Bruxism, Sleep wake disorders.

\section{INTRODUCTION}

Sleep bruxism (SB), a stereotyped oromandibular activity during sleep, is characterized by teeth grinding and clenching [1]. SB was classified in the revised version of the 2005 International Classification of Sleep Disorders as a "sleep-related movement disorder" [2]. Based on bed-partner reports of tooth grinding, SB occurs in 5-8\% of the adult population. The prevalence of SB decreases from childhood at $14-20 \%$ to old age at 3\%. No gender difference in SB prevalence has been noted [3].

The evolution of definition and classification of SB has allowed this entity to become more distinct and homogenous. Increased focus facilitates research activities, resulting in improved understanding of etiological factors and physiological processes associated with SB. Despite many recognized risk factors for SB, its etiology and pathophysiology are yet to be established. There is convincing evidence that bruxism is part of an arousal response and sleep-related movement disorder. In short, bruxism is mainly mediated centrally, not peripherally [4]. SB is no longer considered to be simply related to mechanistic factors such as occlusal discrepancies or due to psychological issues such as stress, anxiety, depression, or a combination of them. SB is considered as primarily a sleep-related movement disorder with a yet to be discerned multifactorial etiology and complex multisystem physiological processes [5].

SB has many clinical symptoms, including a tooth-grinding noise that might be reported by bed partner, pain in the temporomandibular joint and masticatory muscles, headache (especially in the temporal zone when the patient wakes up in the morning), tooth hypersensitivity, and morning jaw stiffness. Some clinical signs include abnormal tooth wear, tongue indentation, linea alba, hypertrophy of masseter muscles, and breakage of fillings and/or teeth. However, many of the deleterious effects of SB are permanent; clinically speaking, it is sometimes difficult to ascertain [6].

\section{MANAGEMENT OF SLEEP BRUXISM}

There are various treatment modalities for the management of SB, including occlusal appli- 
ances, behavioral approaches, and pharmacological management. However, none of them has been reported to be able to treat SB perfectly [2]. Treatment of bruxism is indicated when the disorder causes any of these aforementioned problems. Treatment is designed primarily to prevent further damage to the teeth. Unfortunately, there is no evidence that bruxism can be permanently altered by dental, behavioral, or pharmacological intervention. Clinician's choice of management option is driven by the need to protect orofacial structures from damage and relieve any accompanying pain related to sensory complaints.

\section{Occlusal Approaches}

Oral appliances may reduce bruxism, muscular activities, and myofascial pains. Oral appliances can generally prevent tooth wear and injury and perhaps reduce night time clenching or grinding behavior rather than altering a causative malocclusion. Although these appliances can help reduce muscle pain, they are unlikely to significantly reduce nocturnal behavior. These appliances are worn every night by bruxers (Fig. 1) [7]. They can protect dental surfaces and relax masticatory muscles. They have been extensively used in clinical practice. However, their exact mechanisms of action are still under debate. No evidence supports their role in stopping SB.

\section{Behavioral Approaches}

Nocturnal alarms have been used to treat bruxism. Typically, these devices monitor masticatory electromyography (EMG) activity or sounds. When the activity exceeds a threshold for a given period of time, an alarm sounds and wakes the bruxer to terminate the grinding/clenching behaviors. Because sleep is disrupted, patients who use nocturnal alarms may report sleepiness and difficulty in concentrating if an alarm is being used.

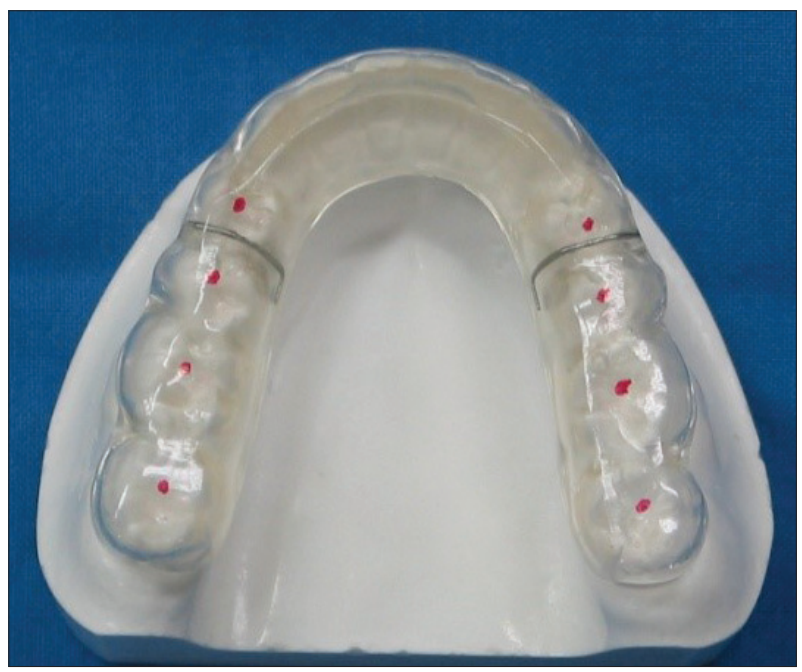

Fig. 1. An occlusal view of oral appliance. Although this appliance can help reduce muscle pain, they do not always stop a patient from bruxing. This appliance is worn every night by bruxer.
Discontinuation of the alarm may lead to "Rebound" in the level of grinding.

\section{Pharmacologic Approaches}

The etiopathophysiology of SB is still unclear. However, considerable evidence indicates that monoamine neurotransmitters, especially dopamine, may play a role in the development of this disorder. A previous controlled study of dopamine precursor L-dopa, and a recent case report involving dopamine D1/ D2 receptor agonist pergolide have demonstrated the effective use of dopaminergic agents for treatment of SB [8]. This supports the currently unproven hypothesis that dopamine depletion causes this disorder [9]. However, inconsistent results have been reported for another D2 agonist, bromocriptine. Medications for the management of bruxism have been studied increasingly over the last few years, including BoNT, anticonvulsant drugs, and selective serotonin reuptake inhibitor. Unfortunately, there is only limited evidence showing efficacy of any of these drugs. Most studies are case reports. Thus, no definite conclusions can be drawn. Despite studies have provided evidence that the dopaminergic system plays a crucial role in SB, the mechanism of psychotropic drug-induced bruxism is still poorly understood. Psychotropic drugs can also modulate other neurotransmitters. This may cause different and sometimes opposite effects on either awake or SB [9].

The most recent advocated pharmacological approach to manage pathological effects of nocturnal and diurnal bruxism is by injecting muscles of mastication with BoNT. Recent research studies have shown that BoNT is effective for controlling involuntary orofacial movements and secondary bruxism in patients with movement disorders (e.g., cranial dystonia) [1013]. However, most relevant studies are case reports. Very few systematic studies have provided high-quality data for the management of SB. Lee et al. [14] have studied the effect of BoNT on nocturnal bruxism using a portable EMG device and found that BoNT injection can reduce the number of nocturnal bruxism events for up to 12 weeks. However, these data were assessed by automatic detections based on EMG threshold. This method has difficulty in discriminating between SB and other oral motor activities based on portable EMG. Although many studies have evaluated SB patients and normal non-bruxers using polysomnography (PSG) $[15,16]$, only one study has used PSG to study the effect of BoNT on SB [17].

It is known that masseter and temporalis muscles are muscles related to SB [17]. Many case studies have reported injections of the masseter muscle, while others have injected both masseter and temporal muscles [18-20]. Although many people might think that the temporal muscle plays a smaller role in SB, it is likely that temporal and masseter muscles are involved in SB equally.

Only one study has evaluated the effect of a single BoNT injection on SB using PSG [17]. Results of that study showed that 
BoNT injection to masseter and temporalis muscles did not change the occurrence of rhythmic masticatory muscle activity (RMMA) episodes or orofacial activities. However, the amplitude of EMG burst during RMMA episodes was significantly decreased for muscles that received BoNT injection. There were no significant differences in usual sleep variables such as sleep efficiency, arousal index, sleep stages, or awakenings per hour between baseline and follow-up recordings [17].

These findings confirmed that the effect of BoNT on jaw motor activity during sleep was characterized by a reduction in the intensity of contractions in injected muscles rather than by a reduction in event occurrence [21-24]. In that study, BoNT at a dose of $25 \mathrm{U}$ was usually injected into each muscles using a 1 -mL-syringe with a 29-gauge, 0.5 -inch needle. BoNT was injected into two to three sites of each subject's masseter and/or temporalis muscles. The method of injection into masseter muscle is the same as the injection for masseter muscle hypertrophy. In the temporalis muscle, the first site was the prominent part of the anterior temporalis muscle that was observed when the subject was asked to clench. It was parallel to the eyebrow. The other two sites were $10 \mathrm{~mm}$ and $20 \mathrm{~mm}$ posterior from the first site [17].

\section{ANATOMICAL RECOMMENDATIONS FOR SAFE BOTULINUM TOXIN INJECTION INTO MASSETER AND TEMPORALIS MUSCLE}

\section{Masseter Muscle}

The injection of the masseter muscle in SB patient use the

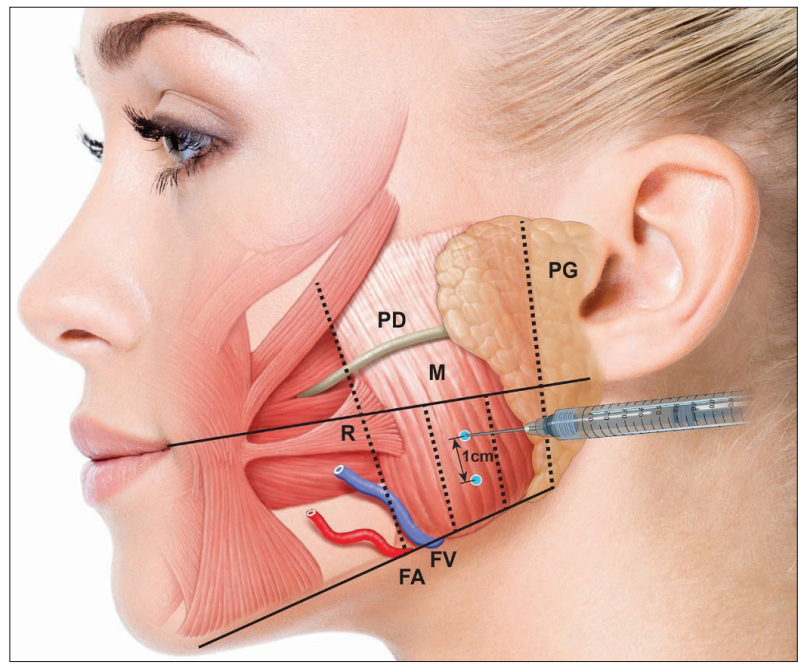

Fig. 2. Botulinum toxin injection sites (blue dots) separated by $1 \mathrm{~cm}$ in the center of the middle third of the masseter muscle. These two injection points are used for most females and for males with a narrow cheek, with $25 \mathrm{U}$ of botulinum toxin being injected. PG: parotid gland, PD: parotid duct, M: masseter muscle, $\mathrm{R}$ : risorius muscle, FA: facial artery, FV: facial vein. same technique as that used for masseteric hypertrophy injection. Botulinum toxin injection sites (blue dots) are separated by $1 \mathrm{~cm}$ in the center of the middle third of the masseter muscle. These two injection points are used for most females and for males with a narrow cheek, with $25 \mathrm{U}$ of botulinum toxin being injected (Fig. 2).

\section{Temporalis Muscle}

Lee et al. [25] have performed a study to simplify optimal temporal areas with a safe and reproducible approach for BoNT injections into the temporalis muscle. They carried out detailed dissections and measurements of structures in the temporal area to virtually represent a topographic mapping of postural

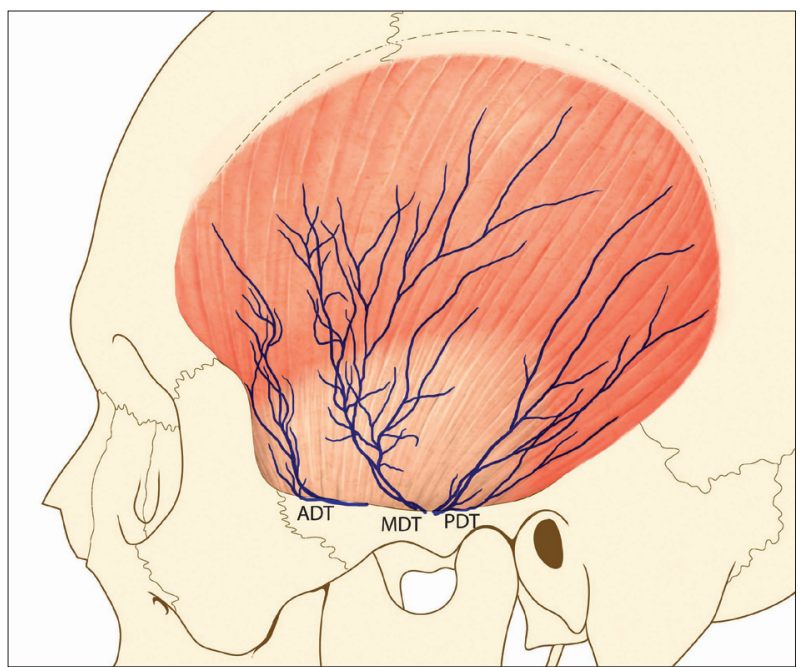

Fig. 3. Lateral view of the neuromuscular partitions of the temporalis muscle. ADT: anterior deep temporal nerve, $\mathrm{M}$ : masseteric nerve, MDT: middle deep temporal nerve, PDT: posterior deep temporal nerve.

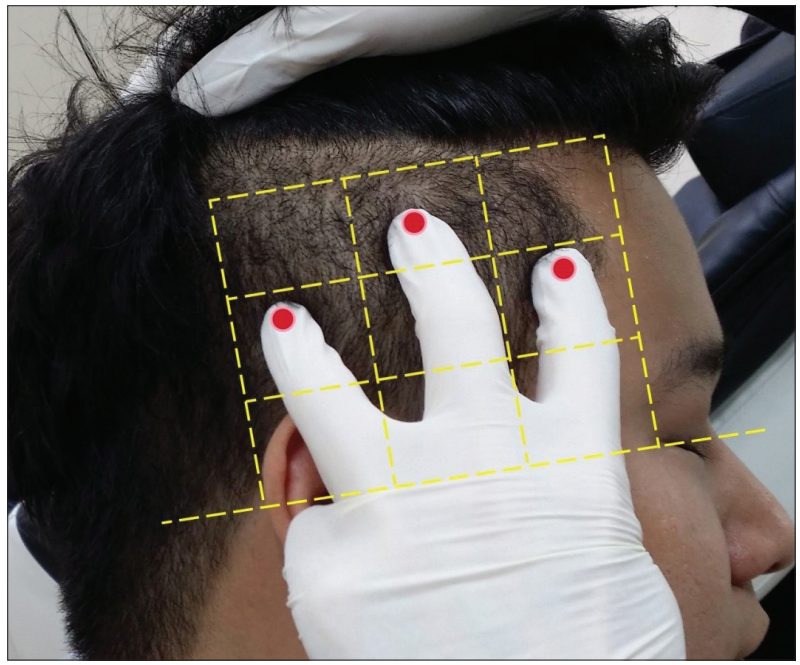

Fig. 4. Suggested temporal injection points for sleep bruxism. It is recommended to inject a total of $25 \mathrm{U}$ of botulinum toxin into three points (red dots on fingertips) within the nine compartments of the temporal region. 
relations among major anatomical structures, such as temporal branch of the facial nerve in the temporalis muscle of the temporal area (Figs. 3 and 4).

They proposed safe and reproducible injection sites in the form of compartments. It would be helpful to further specify the exact position of the injection site within the nine temporalis muscle compartments described herein, to enable clinician to perform injections with greater confidence.

\section{CONCLUSION}

SB and its consequences are complex. They require a committed personalized approach to change behaviors through oral appliances, behavioral approaches, and medications. The primary option for managing SB is still a full-arch oral appliance. For extreme cases, BoNT injections can be applied.

\section{Conflicts of Interest}

The authors have no financial conflicts of interest.

\section{Authors' Contribution}

Conceptualization: Shim YJ. Data curation: Kim SR. Formal analysis: Park KJ. Funding acquisition: Kim ST. Methodology: Kim ST. Project administration: Shim YJ. Resources: Kim SR. Software: Park KJ. Supervision: Kim ST. Validation: Kim ST. Visualization: Park KJ. Writing_original draft: Kim SR. Writing—review \& editing: Shim YJ.

\section{REFERENCES}

1. Bader G, Lavigne G. Sleep bruxism; an overview of an oromandibular sleep movement disorder. Review article. Sleep Med Rev 2000;4:27-43.

2. American Academy of Sleep Medicine. International classification of sleep disorders: diagnostic and coding manual. 2nd ed. Weschesters, IL: American Academy of Sleep Medicine 2005.

3. Lavigne GJ, Montplaisir JY. Restless legs syndrome and sleep bruxism: prevalence and association among Canadians. Sleep 1994;17:739-43.

4. Clarke NG, Townsend GC, Carey SE. Bruxing patterns in man during sleep. J Oral Rehabil 1984;11:123-7.

5. Lavigne GJ, Rompré PH, Poirier G, Huard H, Kato T, Montplaisir JY. Rhythmic masticatory muscle activity during sleep in humans. J Dent Res 2001;80:443-8.

6. Okeson JP. Etiology of functional disturbances in the masticatory system. In: Okeson J. Management of temporomandibular disorders and occlusion. 7th ed. Missouri: Mosby 2011.

7. Okeson JP. Treatment of masticatory muscle disorders. In: Okeson J. Management of temporomandibular disorders and occlusion. 7th ed.
Missouri: Mosby 2011.

8. Lobbezoo F, Lavigne GJ, Tanguay R, Montplaisir JY. The effect of catecholamine precursor L-dopa on sleep bruxism: a controlled clinical trial. Mov Disord 1997;12:73-8.

9. Falisi G, Rastelli C, Panti F, Maglione H, Quezada Arcega R. Psychotropic drugs and bruxism. Expert Opin Drug Saf 2014;13:1319-26.

10. Van Zandijcke M, Marchau MM. Treatment of bruxism with botulinum toxin injections. J Neurol Neurosurg Psychiatry 1990;53:530.

11. Ivanhoe CB, Lai JM, Francisco GE. Bruxism after brain injury: successful treatment with botulinum toxin-A. Arch Phys Med Rehabil 1997; 78:1272-3.

12. Pidcock FS, Wise JM, Christensen JR. Treatment of severe post-traumatic bruxism with botulinum toxin-A: case report. J Oral Maxillofac Surg 2002;60:115-7.

13. See SJ, Tan EK. Severe amphethamine-induced bruxism: treatment with botulinum toxin. Acta Neurol Scand 2003;107:161-3.

14. Lee SJ, McCall WD Jr, Kim YK, Chung SC, Chung JW. Effect of botulinum toxin injection on nocturnal bruxism: a randomized controlled trial. Am J Phys Med Rehabil 2010;89:16-23.

15. Dutra KM, Pereira FJ Jr, Rompré PH, Huynh N, Fleming N, Lavigne GJ. Oro-facial activities in sleep bruxism patients and in normal subjects: a controlled polygraphic and audio-video study. J Oral Rehabil 2009;36:86-92.

16. Lavigne GJ, Rompré PH, Montplaisir JY. Sleep bruxism: validity of clinical research diagnostic criteria in a controlled polysomnographic study. J Dent Res 1996;75:546-52.

17. Shim YJ, Lee MK, Kato T, Park HU, Heo K, Kim ST. Effects of botulinum toxin on jaw motor events during sleep in sleep bruxism patients: a polysomnographic evaluation. J Clin Sleep Med 2014;10:291-8.

18. Hu KS, Kim ST, Hur MS, Park JH, Song WC, Koh KS, et al. Topography of the masseter muscle in relation to treatment with botulinum toxin type A. Oral Surg Oral Med Oral Pathol Oral Radiol Endod 2010;110:16771.

19. Ziccardi VB, Mu L, Schneider RE, Sanders I. Innervation pattern of the temporalis muscle. J Craniofac Surg 1998;9:185-9.

20. Hwang K, Cho HJ, Chung IH. Innervation of the temporalis muscle for selective electrical denervation. J Craniofac Surg 2004;15:352-7.

21. Monroy PG, da Fonseca MA. The use of botulinum toxin-a in the treatment of severe bruxism in a patient with autism: a case report. Spec Care Dentist 2006;26:37-9.

22. Tan EK, Jankovic J. Treating severe bruxism with botulinum toxin. $J$ Am Dent Assoc 2000;131:211-6.

23. Guarda-Nardini L, Manfredini D, Salamone M, Salmaso L, Tonello S, Ferronato G. Efficacy of botulinum toxin in treating myofascial pain in bruxers: a controlled placebo pilot study. Cranio 2008;26:126-35.

24. Watts MW, Tan EK, Jankovic J. Bruxism and cranial-cervical dystonia: is there a relationship? Cranio 1999;17:196-201.

25. Lee WK, Bae JH, Hu KS, Kato T, Kim ST. Anatomical recommendations for safe botulinum toxin injection into temporalis muscle: a simplified reproducible approach. Surg Radiol Anat 2017;39:263-9. 